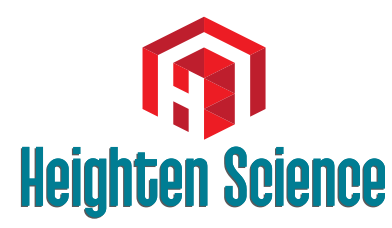

P U B L I C I T I O N S Corporation

\title{
Linear IgA bullous dermatosis in a child successfully responding to oral antibiotics
}

\author{
G Senhaji*, H Bay Bay, R Dassouli, O El Jouari, A Lamouaffaq, \\ Z Douhi, S Elloudi and FZ Mernissi \\ Dermatology department, University Hospital Hassan II, Fez, Morocco
}

*Address for Correspondence: Dr. Senhaji Ghita, Department of Dermatology, University Hospital Hassan II, Fez, Morocco, Tel: 212 600075857; Email: ghitasenhaji88@gmail.com

Submitted: 15 November 2018

Approved: 05 December 2018

Published: 06 December 2018

Copyright: (c) 2018 Senhaji G, et al. This is an open access article distributed under the Creative Commons Attribution License, which permits unrestricted use, distribution, and reproduction in any medium, provided the original work is properly cited

Keywords: Linear IgA bullous dermatosis; Autoimmune bullous dermatosis;

Subepidermal blisters; Antibiotics

Check for updates

\section{Abstract}

Linear IgA bullous dermatosis (LABD) is a rare, chronic, autoimmune bullous dermatosis affecting young children and adults. The exact pathogenesis of this disease is still unknown, although both humoral and cellular immune response are involved. Clinically, it may show heterogeneous skin manifestations. However, it is characterized histologically by linear immunoglobulin A $(\operatorname{lgA})$ deposits over the basal membrane, causing subepidermal blisters. Studies on LABD are relatively sparse and most of the publications are small series or single case reports. Several treatments are reported in literature, however, they should be used with care due to the risk of side effects. We report a case of linear IgA dermatosis with generalized lesions in a 7 year old child, with good outcome under dermocorticoids and antibiotics.

\section{Introduction}

Linear IgA bullous dermatitis (LABD) is a rare, chronic, autoimmune bullous dermatosis affecting young children and adults [1]. It is characterized by linear immunoglobulin A (IgA) deposits over the basal membrane, causing sub epidermal blisters [2]. Studies on LABD are relatively sparse and most of the publications are small series or single case reports [1]. Additionally, In spite of its good prognosis, most children need treatment to reduce the severity of the eruption and to shorten its duration [2]. Thus, considering the rarity of this disease and the importance of the dermatologist's awareness of it for early diagnosis [3], we report a case of linear IgA dermatosis with generalized lesions in a 7 year old child, with good outcome under dermocorticoids and antibiotics.

\section{Case Report}

A 7-year-old child, having parents from a first-degree consanguineous marriage, followed since age 2 for deafness with recurrent episodes of tonsillitis and pharyngitis, for whom he had undergone a tonsillectomy with placement of ear implants. He was also followed up for a productive chronic cough with bronchial dilatation on thoracic $\mathrm{CT}$, and he was complaining of a chronic fluid diarrhea since age 2 with marked weightloss delay. Furthermore, he had a family history of atopy and asthma. The patient complained since birth of a chronic moderate pruritus that had become diffuse and more marked later, aggravated at 2 years of age by the appearance of very itchy fluidlike lesions appearing first at the axillary and genital folds with latter extension on the trunk, back, limbs and face evolving in a context of apyrexia. Clinical examination showed multiple erosions with clear content vesicles grouped in bouquet and rosette, surmounted by places of hemorrhagic crusts, on the face, especially in the perioral and retro auricular areas, on the trunk, limbs, buttocks and scalp without involvement 
of palmoplantar regions (Figure 1). Additionally, there were diffuse cicatricial hypochromic macules with a marked cutaneous xerosis. Somatic examination showed no more abnormalities. A cutaneous biopsy was performed, with a histology in favor of a linear IgA dermatosis (Figures 2,3), and a negative DIF. The patient was tested for immune deficiency, celiac disease and cystic fibrosis with negative results. The diagnosis of linear IgA dermatosis was retained and the patient was treated by oral antibiotics with penicillin A at a dose of $50 \mathrm{mg} / \mathrm{kg} /$ day for a long term. Other associated measures included the use of emollients, oral antihistamines and potent dermocorticoids. The patient experienced a good clinical progress with regression of both lesions and pruritus under oral antibiotics.

\section{Discussion}

LABD is a rare autoimmune vesiculobullous disease with an incidence of 0.2 to 2.3 cases per million-population per year [4]. There are 2 variants: LABD of the adult and of the child, often called chronic bullous disease of childhood [5]. In the later, the disease develops after six months of age, and shows incidence peaks in preschool children, as in our case [6]. Although both humoral and cellular immune response are involved [4]. In fact, it is speculated that IgA autoantibodies produces chemotaxis of eosinophils and basophils that degranulates releasing their lysosomes, which is responsible for the formation of the subepidermal blisters [2]. However, while most cases of LABD are idiopathic, it can be induced by several drugs, mostly vancomycin [4], or non-steroidal anti-inflammatory agents. Some cases can also be preceded by upper respiratory infections [3].

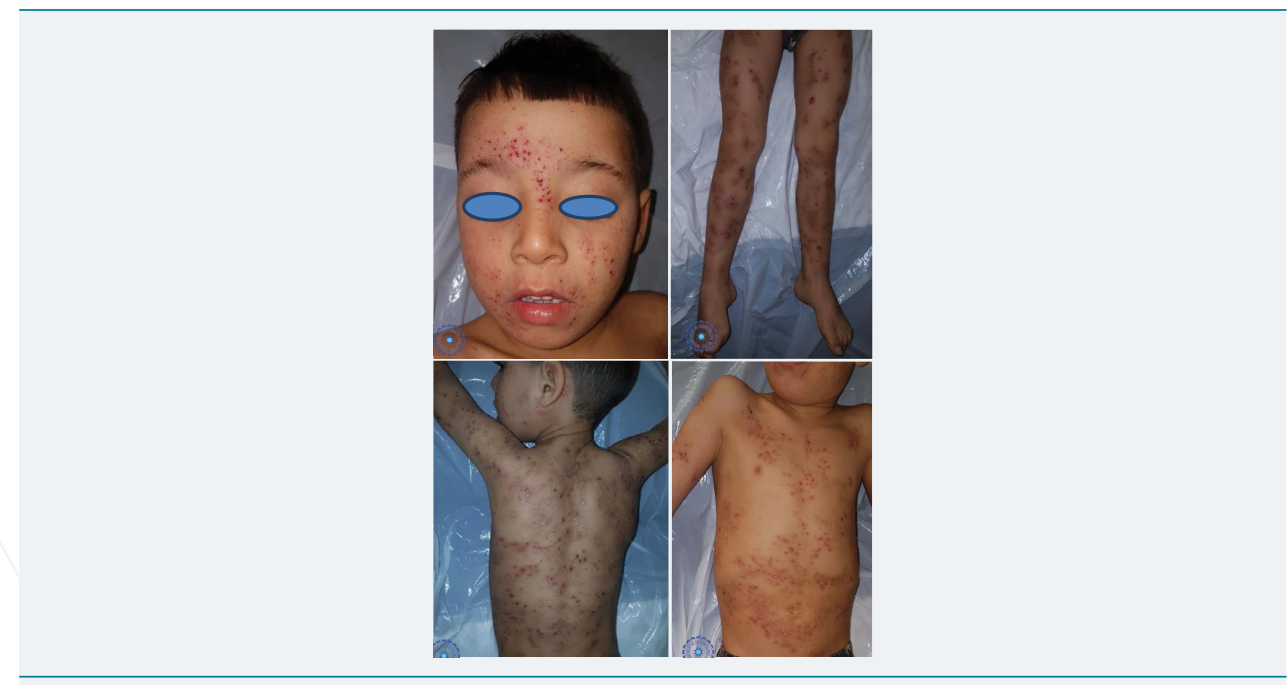

Figure 1: Clinical images showing multiple widespread erosions with clear content vesicles grouped in bouquet and rosette, surmounted by places of hemorrhagic crusts, on the face, trunk, limbs and scalp.

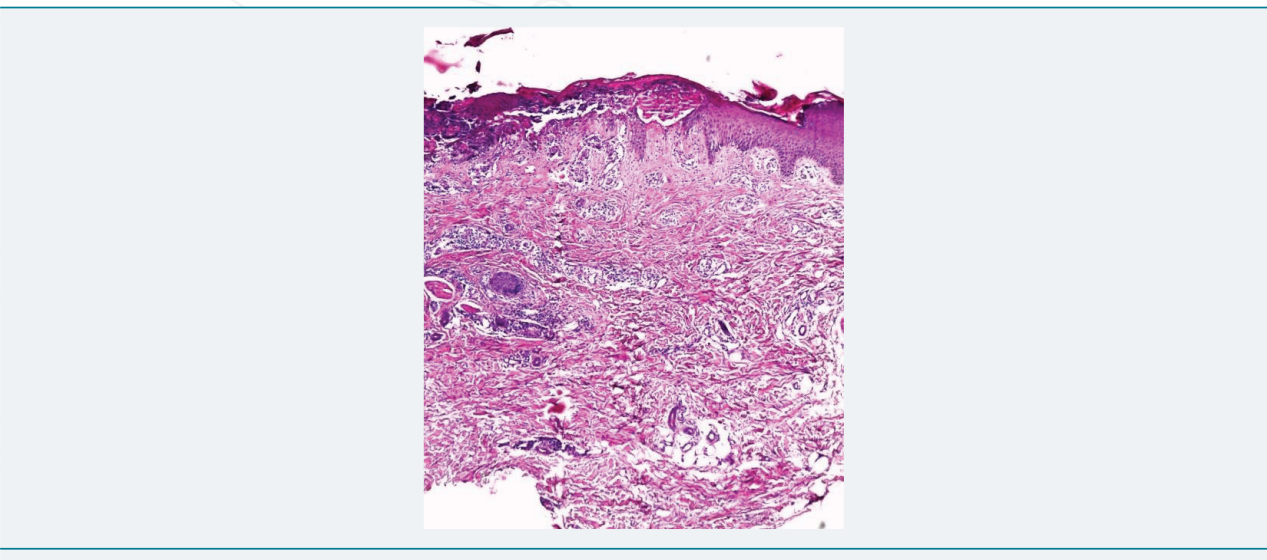

Figure 2: Histological section showing a psoriasiform and slightly spongiotic dermatosis, with zone of excoriation and subepidermal detachment. (HES staining, $x 50$ ). 


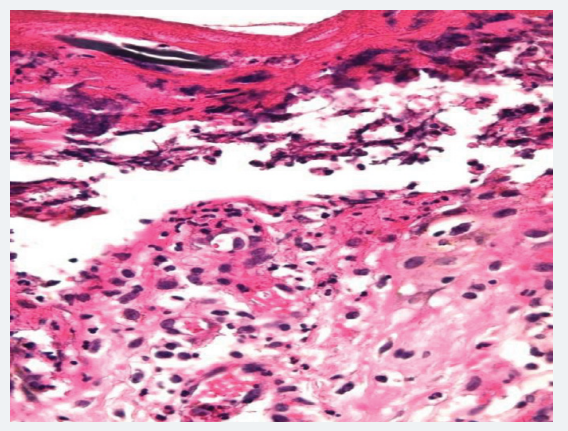

Figure 3: Histological section showing a sub epidermal blister with margination of neutrophils at the top of some dermal papillae. (HES staining, $x$ 400).

Clinically, it may show heterogeneous skin manifestations [5]. Polycyclic groupings of bullae with central crusting, called 'cluster of jewels' or 'string of pearls' is the most characteristic feature, especially in childhood spontaneous forms [7]. The bullae can rupture due to intense itching and resolve as crusts and erosions [4]. This was seen in our patient which clinical examination showed multiple erosions with clear content vesicles grouped in bouquet and rosette and hemorrhagic crusts by places. There may also be erythematous papules, urticarial plaques, or vesicobullous lesions with annular or arciform configuration when new bullous lesions develop on the periphery of previous lesions $[3,8]$. Subjective symptoms are usually present, and can vary from almost none or mild pruritus to severe burning [1]. Our patient experienced chronic pruritus which is the main symptom reported in the literature. LABD lesions typically have a widespread distribution, most notably on the thighs, buttocks, lower trunk, genital region, scalp, and face [4]. Mucosal involvement can also be seen, especially in the oral and ocular regions, with a pattern of lesions similar to patients with cicatriciel pemphigoid [6].

LABD has been reported in association with systemic diseases, such as autoimmune and autoinflammatory conditions like ulcerative colitis, Crohn's disease, rheumatoid arthritis and psoriasis [4]. It has also been described in association with malignancy [1].

On histopathological examination, there is a subepidermal blister formation with a predominaly neutrophils along the basement membrane zone, characteristically arranged in a linear array, as seen in our case [5]. However, immunofluorescence studies are very important for the diagnosis of linear IgA bullous dermatosis of childhood, and constitute the gold standard [4]. Direct immunofluorescence (DFI) typically confirms a diagnosis by showing a linear and homogeneous IgA deposition along the BMZ $[1,6]$. However, there may also rarely be coexistence of IgG, IgM, and C3 [4]. Nevertheless, DIF may show negative in some cases, as in our patient, without eliminating the diagnosis.

The main differential diagnosis is dermatitis herpetiformis, which differs by granular IgA deposits along the basement membrane zone [5], among with bullous pemphigoid of the childhood, that shares similar clinical and histopathological characteristics [6]. Other differential diagnosis includes bullous impetigo, epidermolysis bullosa acquisita and scabies [4].

Several treatments are reported in literature, with dapsone being the first-line treatment [8]. However, it should be used with care, due to the risk of side effects [6], mainly methemoglobinemia and hemolytic anemia [3]. Monotherapy with dapsone is usually sufficient in many cases, but the association with corticosteroids and immunosuppressants such as azathioprine and cyclophosphamide may be necessary in more severe cases or to limit its side effects $[3,5]$. Furthermore, some antimicrobials, such as erythromycin and dicloxacillin have also been found to be highly effective especially 
in mild cases $[4,6]$. Their benefical effects in LABD may be due to their anti-inflammatory effects through inhibition of neutrophil chemotaxis and release of Lysozymes [2]. Moreover, once the disease is controlled, the minimum dose of medication is required to control the symptoms [6]. In our case, we opted for a treatment based on antibiotics for their proven effectiveness in previous publications and in order to avoid the multiple side effects of heavy treatments like dapsone on a child of this age.

Owing to the rarity of LABD and the lack of long-term patient follow-up, the natural disease course remains underdescribed. However, most cases in children spontaneously resolve within two years, or may persist until puberty [6].

\section{Conclusion}

In conclusion, as this rare disease can present in such a heterogenous and nonclassical fashion, it is important to make the correct diagnosis of LABD, based on the clinical and immunological findings, and to eliminate other confusing diagnosis like dermatitis herpetiform and bullous pemphigoid, in order to provide the optimum treatment and care for the patient [1,3,]. Additionally, as seen in our case, we believe that therapy with antibiotics, because of its low index of side-effects in paediatric patients, should be the first step in the treatment, as the clinical response can be rapid and long-lasting for pediatric case [2].

\section{References}

1. Lings K, Bygum A. Linear IgA Bullous Dermatosis: A Retrospective Study of 23 Patients in Denmark. Acta Derm Venereol. 2015; 95: 466-471. Ref.: https://goo.gl/nN4mZG

2. De las Heras MN. Linear IgA bullous dermatosis of childhood: good response to antibiotic treatment. Clin Exp Dermatol. 2014; 39: 395-397. Ref.: https://goo.gl/zN6fci

3. Machado TYS, Enokihara MMSS, lida TM, Porro AM. Adult linear IgA bullous dermatosis: report of three cases. An Bras Dermatol. 2018; 93: 435-437. Ref.: https://goo.gl/7uuDa1

4. Chaudhari S, Mobini N. Linear IgA Bullous Dermatosis A Rare Clinicopathologic Entity with an Unusual Presentation. J Clin Aesthet Dermatol. 2015; 8: 43-46. Ref.: https://goo.gl/9Zf9KK

5. Gouveia Al, Teixeira A, Freitas JP, Soares-de-Almeida L, Filipe P, et al. Linear Immunoglobulin A Bullous Dermatosis. J Pediatr. 2016; 170: 338-338. Ref.: https://goo.gl/m2E7Gh

6. Fernandes KAP, Galvis KH, Gomes ACMS, Nogueira OM, Felix PAO, et al. Linear IgA and IgG bullous dermatosis. An Bras Dermatol. 2016; 91 (5 Supl 1): S32-34. Ref.: https://goo.gl/kuyAF8

7. Chanal J, Ingen-Housz-Oro S, Ortonne N, Duong TA, Thomas M, et al. Linear IgA bullous dermatosis: comparison between the drug-induced and spontaneous forms. Br J Dermatol. 2013; 169: 10411048. Ref.: https://goo.gl/oQY68Q

8. Ferreira $\mathrm{H}$, Vilarinho $\mathrm{C}$. Linear IgA dermatosis: An atypical manifestation of a rare disease. Pediatr Neonatol. 2018; 59: 324-325. Ref.: https://goo.gl/Fg2GxP 